\title{
Games with a scientific purpose
}

\author{
Benjamin M Good and Andrew I Su*
}

\section{Abstract \\ The protein folding game Foldit shows that games are an effective way to recruit, engage and organize ordinary citizens to help solve difficult scientific problems.}

Modern science is filled with challenges of massive scale. From classifying terabytes of astronomical images to annotating gigabases of genome sequence, many of these challenges require enormous amounts of human effort. To address these kinds of labor-intensive problems, scientists have increasingly sought to harness the voluntary contributions of large communities of individuals from outside the traditional scientific community. These crowdsourcing initiatives work by distributing the required cognitive labor across the minds of thousands and sometimes millions of volunteers, mirroring how the Search for Extraterrestrial Intelligence (SETI) project first distributed computational tasks across many of the world's personal computers. This general strategy has been used successfully, for example, to classify the morphologies of uncharted galaxies [1], to conduct comprehensive ecological censuses [2], and to organize knowledge around human gene function [3]. The key challenge for these efforts to succeed is to inspire the required numbers of volunteers.

The motivations for individuals to voluntarily contribute to crowdsourcing initiatives have been widely debated, but they range from altruism, to ego, to a shared sense of purpose. One emerging trend expands that list of motivations to include the pursuit of fun and enjoyment through games. In particular, David Baker and colleagues [4] have described several success stories behind their game for protein folding, called Foldit. Among these successes, the most recent and perhaps the most groundbreaking was the development of a novel algorithm for protein folding by game players.

*Correspondence: asu@scripps.edu

Department of Molecular and Experimental Medicine, The Scripps Research Institute, La Jolla, CA 92037, USA

\section{Making protein folding fun}

Gamers aren't just trying to win games anymore. They have a bigger mission. They're on a mission to be a part of something epic. [5]

In many of the most successful video games, the players' basic mission is the same: to save the world. Whether it is alien invaders or zombie-creating viruses, the imminent peril of the human race is at hand and you are the only one who can stop it. Foldit harnesses this same instinct to motivate players in a similarly epic mission, specifically to unlock the secrets of protein folding.

In its implementation, Foldit is a visual puzzle game. The player is presented with a primary protein sequence or partially folded structure, and then challenged to find its lowest-energy three-dimensional structure [6]. Players interactively manipulate the protein structure by pulling, twisting and tugging the protein backbone and side chains into various conformations. Foldit also provides players with a panel of tools that automate common operations, such as 'wiggle', which performs a local gradientbased energy minimization.

Foldit gives continuous feedback to the player about the quality of their folding attempts through a real-time score derived from the Rosetta free energy function. As more hydrophobic residues are buried and more hydrogen bonds established, the player's point total increases. In addition, the interface provides visual cues that highlight, for example, steric clashes that need to be resolved. The process of manually folding the protein provides players with the visual satisfaction of solving a puzzle, which, in addition to improving their numeric score, provides some of the psychological rewards of gameplay.

Foldit is also a social game. Using the website and provided software, players can share their partially solved puzzles and folding strategies, chat live with other players and contribute to a wiki for the Foldit community. Players can also join teams, participate in contests and track their progress on leaderboards.

\section{From structure solution to algorithm discovery}

The scientific value of the Foldit system was first demonstrated by showing that game players could solve specific 
structure prediction problems. In the first major publication to discuss Foldit, Baker and colleagues [6] showed that game players could, in many cases, generate better structure predictions than the state-of-the-art Rosetta structure prediction program. Foldit then unleashed their army of folders on the task of solving the structure of the Mason-Pfizer monkey virus retroviral protease, a problem that was previously intractable to both computational and experimental methods [7]. After 3 weeks of game play, the best solutions were screened and, remarkably, a solution to this previously unsolved structure was identified and subsequently validated. This achievement established Foldit as a legitimate resource for the structural biology community.

Although solving individual structures is valuable, an even greater challenge is to develop the ability to solve any structure automatically. Accordingly, Baker and colleagues [4] recently tested the gamer community's proficiency at actually designing protein folding algorithms. Foldit provides players with a script-building interface that they can use to design and execute their own folding 'recipes'. Recipes can be constructed using either a scripting language or a graphical interface that allows players to drag and drop different folding actions together into a workflow. These recipes can be shared with and extended by other players in much the same manner that code from open source software projects can be mixed and matched by developers.

During a 3.5 month study period, 568 Foldit players wrote 5,202 folding recipes, which were collectively executed more than 150,000 times. Sharing between players resulted in the social evolution of the best strategies. In particular, a single strategy named 'Blue Fuse' ultimately came to dominate the recipe landscape, being used more than three times as often as any other strategy. On detailed examination, Blue Fuse bore a strong resemblance to an unpublished algorithm called 'Fast Relax', which was concurrently being developed by members of the Baker laboratory. Applying both algorithms within the computational constraints of the Foldit environment, Blue Fuse showed superior performance to the algorithm developed by professional structural biologists.

Although the final author of the Blue Fuse recipe provided the crucial adaptations that made it successful, these would not have been possible without the work of other members of the community. Effectively, the gamer community collaboratively wrote the Blue Fuse algorithm using Foldit as a distributed software development environment. Baker and colleagues [4] noted that Blue Fuse was an adaptation of another player's algorithm, 'Acid Tweaker v0.5', and that Blue Fuse itself has subsequently been adapted into many different forms by many different players. Although the early successes of the Foldit initiative highlighted the game's ability to tap into human spatial reasoning skills, the task of developing folding algorithms is dependent on a much broader and less common skill set. By demonstrating that games can be used to motivate contributions of this level of complexity, Foldit has again expanded the scope of what might be possible with scientific games.

\section{The untapped potential}

Foldit has proved to be a useful new tool in the study of protein folding. Using games to tap into the wisdom of crowds has resulted in tangible advances. It is intriguing to consider whether games can be productively used in other areas of biology, including genomics. For example, might it be possible to build a game out of predicting phenotype from genotype?

In addition to expanding the scientific scope of games, there is also the opportunity to substantially expand the size of the scientific gamer population. Foldit has attracted almost 230,000 players since its release. What is most remarkable is not how massive this figure is, but how much more massive it could become. It is estimated that hundreds of millions of gamers worldwide collectively spend more than 3 billion hours per week playing video games [5]. If just $1 \%$ of that effort - now spent on pursuits such as Solitaire, World of Warcraft and Angry Birds - could regularly be applied to purposeful scientific games, we could accomplish all of the work conducted by Foldit players in the past 3 years many times every single week.

\section{Scientific discovery, or scientist discovery?}

Most crowdsourcing initiatives, including other so-called 'games with a purpose' [8], work by decomposing one massive project into a very large number of simple tasks, which are then distributed among a very large population of participants. For example, the ESP Game (named for the apparent extrasensory perception involved in gameplay) succeeded in producing labels for millions of images by distributing this task over tens of thousands of game players [9]. For crowdsourcing projects like this, nearly any volunteer can add a tangible, even if small, contribution with a minimal amount of training. And each incremental contribution gets the entire project incrementally closer to the overall goal.

However, the challenge of protein folding is neither simple nor easily decomposable. Whether it is presented in the context of a game or not, writing an algorithm for solving protein structures is a hard problem whose solution requires both problem-solving talent and perseverance.

Therefore, the developers of Foldit developed a qualitatively different type of crowdsourcing project. Foldit uses a game to recruit a large number of participants as a mechanism to identify players with exceptional skills. Its large player community and well-defined scoring system 
allow Foldit to identify the relatively few players that can make tangible contributions to the scientific goals. This unique and unprecedented group of gamer-scientists is made up of highly experienced problem solvers with extremely diverse backgrounds - an ideal recipe for creative group problem solving. Without Foldit, this talent and energy would probably have been used on less valuable tasks - such as ridding a game world of zombies.

Zoran Popović, one of the founders of the Foldit project, has a truly audacious ambition for their community of game players:

Our ultimate goal is to have ordinary people play the game and eventually be candidates for winning the Nobel Prize. [10]

Whether or not Foldit eventually empowers one of its game players to win science's highest prize, it is clear from these recent studies that purposefully designed games can help advance scientific progress. And that is epic indeed.

Published: 28 December 2011

\section{References}

1. Lintott CJ, Schawinski K, Slosar A, Land K, Bamford S, Thomas D, Raddick MJ, Nichol RC, Szalay A, Andreescu D, Murray P, van den Berg J: Galaxy Zoo: morphologies derived from visual inspection of galaxies from the Sloan Digital Sky Survey. Mon Not Roy Astron Soc 2008, 389:1179-1189.
2. Sullivan BL, Wood CL, lliff MJ, Bonney RE, Fink D, Kelling S: eBird: A citizenbased bird observation network in the biological sciences. Bio/ Conserv 2009, 142:2282-2292.

3. Huss JW, Orozco C, Goodale J, Wu C, Batalov S, Vickers TJ, Valafar F, Su Al: A gene wiki for community annotation of gene function. PLOS Bio/ 2008, 6:e175.

4. Khatib F, Cooper S, Tyka MD, Xu K, Makedon I, Popovic Z, Baker D, Players F: Algorithm discovery by protein folding game players. Proc Natl Acad Sci USA 2011, 108:18949-18953.

5. McGonigal J: Reality is Broken: Why Games Make Us Better and How They Can Change the World. New York: Penguin; 2011.

6. Cooper S, Khatib F, Treuille A, Barbero J, Lee J, Beenen M, Leaver-Fay A, Baker D, Popovic Z, Players F: Predicting protein structures with a multiplayer online game. Nature 2010, 466:756-760.

7. Khatib F, Dimaio F, Cooper S, Kazmierczyk M, Gilski M, Krzywda S, Zabranska H, Pichova I, Thompson J, Popovic Z, Jaskolski M, Baker D: Crystal structure of a monomeric retroviral protease solved by protein folding game players. Nat Struct Mol Biol 2011, 18:1175-1177.

8. Ahn LV, Dabbish L: Designing games with a purpose. Commun ACM 2008, 51:58-67.

9. Ahn Lv, Dabbish L: Labeling images with a computer game. Proceedings of ACM CHI 2004 Conference on Human Factors in Computing Systems: April 24-29, 2004; Vienna, Austria. Edited by Dykstra-Erickson E, Tscheligi M. ACM Press; 2004:319-326

10. Foldit: Competitive Protein Folding for Medical Science [http://www.engr washington.edu/facresearch/highlights/cse_foldit.html]

doi:10.1186/gb-2011-12-12-135

Cite this article as: Good BM, Su Al: Games with a scientific purpose.

Genome Biology 2011, 12:135. 